\title{
Organophosphate Urinary Metabolite Levels during Pregnancy, Delivery and Postpartum in Women Living in Agricultural Areas in Thailand
}

\author{
Pornpimol KongtiP ${ }^{1}$, Noppanun Nankongnab ${ }^{1}$, Susan WoskiE $^{2}$, Akkarat PhamonPhon ${ }^{1}$, \\ Prapin Tharnpoophasiam ${ }^{3}$, Kitsiluck Wilaiwan ${ }^{4}$ and Punnee Srasom ${ }^{5}$ \\ ${ }^{1}$ Department of Occupational Health and Safety, Faculty of Public Health, Mahidol University, Thailand, ${ }^{2}$ Department \\ of Work Environment, University of Massachusetts Lowell, USA, ${ }^{3}$ Department of Social and Environmental \\ Medicine, Faculty of Tropical Medicine, Mahidol University, Thailand, ${ }^{4}$ Paholpolpayuhasena Hospital, Thailand \\ and ${ }^{5}$ Amnatcharoen Hospital, Thailand
}

\begin{abstract}
Organophosphate Urinary Metabolite Levels during Pregnancy, Delivery and Postpartum in Women Living in Agricultural Areas in Thailand: Pornpimol KongtIP, et al. Department of Occupational Health and Safety, Faculty of Public Health, Mahidol University, Thailand-Objective: Prenatal exposure to organophosphate pesticides can lead to developmental neurotoxicity. A longitudinal birth cohort was established to investigate pesticide exposures from different agricultural activities. Maternal urinary organophosphate metabolites were measured at 28 weeks of pregnancy $(n=86)$, delivery $(n=67)$ and 2 months postpartum $(n=51)$. Method: Subjects were interviewed with questionnaires about work, home and behavioral factors potentially associated with pesticide exposures, and spot urine samples were also collected. The urine samples were analyzed for dimethyl phosphate (DMP), diethyl phosphate (DEP), diethyl thiophosphate (DETP) and diethyl dithiophosphate (DEDTP), using gas chromatography-mass spectrometry. Results: The urinary DMP and dialkyl phosphate (DAP) concentrations at 28 weeks of pregnancy and delivery were not significantly different, but the DMP and DAP concentrations at 28 weeks of pregnancy and DAP concentrations at delivery were significantly different $(p<0.05)$ from those at 2 months postpartum. The factors influencing the urinary DAP concentrations at 28 weeks of pregnancy included insecticide used in the home, living close to agricultural farmland, frequency of agricultural field visits during the first and second trimesters of pregnancies, occupation of subjects, pesticide used and other agricultural
\end{abstract}

Received Feb 22, 2013; Accepted Jun 20, 2013

Published online in J-STAGE Jul 26, 2013

Correspondence to: P. Kongtip, Department of Occupational Health and Safety, Faculty of Public Health, Mahidol University, 420/1 Rajvithi Road, Bangkok 10400, Thailand (e-mail: phpkt@mahidol. ac.th) activities. Conclusions: The urinary organophosphate metabolites, DMP, DEP, DETP, DEDTP, total DEP and DAPs, at 28 weeks of pregnancy, delivery and 2 months postpartum fluctuated depending on their pesticide exposures both at home and in agricultural fields.

(J Occup Health 2013; 55: 367-375)

Key words: Agricultural activities, Organophosphate metabolites, Pesticides, Pregnant women

In Thailand, approximately $40 \%$ of the land is used for agriculture, and $38.7 \%$ of the total national workforce is employed in the agricultural sector ${ }^{1)}$. The Ministry of Agricultural and Cooperatives reported that Thailand imported approximately 39,634 tons of pesticides in 1997 and that the volume increased to 117,815 tons in $2010^{2}$. A survey of intelligence level in Thai children found that the average IQ fell steadily over the years 1997 to 2009 from 91 to 88, which was lower than the WHO standard range of $90-110^{3)}$. The results of the age-appropriate development survey in children under 5 years old conducted by the Department of Health concluded that development of Thai children decreased from $72 \%$ in 2004 to $67 \%$ in $2007^{3)}$. The survey results suggest that poor environment, education and food contributed to the decreasing IQs.

One large birth cohort of primarily Latina women in an agricultural community in the United States was used to examine the association between organophosphate pesticide exposures during pregnancy and the effects on the neurodevelopment of infants and children. The pregnant woman may have been exposed to occupational pesticides or common household pesticides; however, significant impacts on child neurodevelopment were found ${ }^{4-6}$. As part of that study, 
Bradman et al. presented the urinary organophosphate metabolite levels of pregnant women in different periods (13 weeks of gestation, 26 weeks of gestation and 9 days after delivery) ${ }^{7}$. The level of organophosphate metabolites was increased during pregnancy and higher in the postpartum period.

Due to the neurotoxic vulnerability of the developing fetus, exposure of pregnant women to pesticides is of great concern ${ }^{8)}$. Previous studies have shown that in Thailand, there are many cases of improper management of pesticides, inappropriate or nonexistent use of personal protective equipment, use of higher pesticide concentrations than specified on the product label and high volumes of pesticide use on farms ${ }^{9,10)}$. The aims of this study were to investigate the levels of exposure during pregnancy and postpartum and to identify the factors that increase the risk of higher levels of exposure among our cohort.

\section{Subjects and Methods}

\section{Recruitment of pregnant women}

Pregnant women who came for prenatal care at the three hospitals, Amnatchareon Hospital in Amnatchareon Province in the north east, Sawanpracharak Hospital in Nakhonsawan Province in the lower north and Paholpolpayuhasena Hospital in Kanchanaburi Province in the west of Thailand, were recruited during May 2011 to January 2012. To be recruited, the woman had to be in their 7 th month of pregnancy, had to be 20-35 years of age, had to not have diabetes or hypertension and had to be planning to give birth and have follow-up infant care at the recruiting hospital. This study was approved by the Committee on Human Rights Related to Human Experimentation, Faculty of Public Health, Mahidol University, and the University of Massachusetts Lowell Institutional Review Board.

\section{Data collection}

At the hospital, the women were interviewed by nurses regarding general health, diet, work exposures including agricultural work and use of pesticides at home and work at $28^{\text {th }}$ weeks of pregnancy and 2 months postpartum. Spot urine samples were collected in polyethylene bottles at the $28^{\text {th }}$ week of pregnancy, delivery and 2 months postpartum. The urine specimens were stored at $-45^{\circ} \mathrm{C}$ until analysis.

\section{Analysis of urine samples}

The analysis of urinary dialkyl phosphates (DAPs) was modified from Alwis et al. ${ }^{11)}$. Four DAPs were determined: including dimethyl phosphate (DMP) and three diethyl phosphates, diethyl phosphate (DEP), diethyl thiophosphate (DETP), diethyl dithiophosphate (DEDTP).

\section{The urinary DAP analysis method}

Two milliliters of urine was placed in a $10-\mathrm{m} l$ screw-cap tube and spiked with $25 \mu l$ of the internal standard (dibutyl phosphate, $5 \mathrm{mg} / \mathrm{l}$ ), acidified with $3 \mathrm{M}$ hydrochloric acid $(50 \mu l)$ and mixed. SPE cartridges (Bond Elut PPL $500 \mathrm{mg} / 3 \mathrm{ml}$, Varian, Palo Alto, CA, USA) were placed on Visiprep ${ }^{\mathrm{TM}}$ DL Vacuum Manifolds (Supelco, Bellefonte, PA, USA). SPE cartridges were conditioned with acetonitrile $(4 \mathrm{ml})$ followed by $0.1 \mathrm{~mol} / l \mathrm{HCl}(4 \mathrm{ml})$. The urine sample was loaded onto the SPE cartridge at a flow rate of $0.25 \mathrm{~m} / / \mathrm{min}$. The cartridge was dried using nitrogen gas ( 30 psi) for 10 minutes, washed with $0.1 \mathrm{~mol} / l \mathrm{HCl}(1 \mathrm{ml})$, and then dried again for 5 minutes. Elution was accomplished with acetonitrile $(6 \mathrm{ml})$ at a flow rate of $0.5 \mathrm{ml} / \mathrm{min}$ into a $10-\mathrm{m} l$ screw-cap tube containing potassium carbonate ( $25 \mathrm{mg}$ ). The eluate was evaporated to dryness in a Reacti-Therm $^{\mathrm{TM}}$ III Heating Module (Thermo Fisher Scientific Inc., Waltham, MA, USA) with high purity nitrogen for approximately 2 hours. The dried residue was resuspended in $2 \mathrm{~m} l$ of acetonitrile. Potassium carbonate $(\sim 25 \mathrm{mg})$ and $2,3,4,5,6$-Pentafluorobenzyl bromide $(30 \mu l)$ were added to the tube, which was then placed in an oven at $60^{\circ} \mathrm{C}$ for 4 hours. After heating, the sample tubes were allowed to cool to room temperature. The top layer was carefully transferred using Pasteur pipettes to $10-\mathrm{m} l$ centrifuge tubes without disturbing the sediment at the bottom of the tubes. The solutions were evaporated to dryness in a Reacti-Therm $^{\mathrm{TM}}$ III Heating Module (Thermo Fisher Scientific Inc., Waltham, MA, USA) with nitrogen for 30 minutes. The residue was dissolved with $150 \mu l$ of toluene and transferred to an auto-sampler vial for analysis by gas chromatography-mass spectrometry (GC-MS) (Agilent Technologies, Santa Clara, CA, USA). An aliquot $(1 \mu l)$ of the sample was injected in the splitless mode onto a DB-5MS ([5\%-phenyl]methylpolysiloxane) capillary GC column $(30 \mathrm{~m}$, $0.25-\mathrm{mm}$ i.d., $0.25 \mu \mathrm{m}$ ) using the autosampler. The carrier gas used was helium. The injection port and transfer line were set at $250^{\circ} \mathrm{C}$ and $280^{\circ} \mathrm{C}$, respectively. Constant flow mode was used with a flow rate of $1.2 \mathrm{ml} / \mathrm{min}$. The temperature of the $\mathrm{GC}$ oven was initially set at $90^{\circ} \mathrm{C}$ for 1 minute, increased at $4^{\circ} \mathrm{C} / \mathrm{min}$ to $150^{\circ} \mathrm{C}$ and then increased at $50^{\circ} \mathrm{C} / \mathrm{min}$ to $270^{\circ} \mathrm{C}$. The final temperature of $270^{\circ} \mathrm{C}$ was held for 5 minutes. The MS was operated in electron impact ionization mode at $70 \mathrm{eV}$, and ions were monitored in the selected ion monitoring mode.

The calibration curves of four dialkyl phosphate (DAP) metabolites were prepared at concentrations of $1,5,10,25,50,75$ and $100 \mathrm{ng} / \mathrm{ml}$. The ranges of urinary DMP, DEP, DETP and DEDTP in field samples were not detectable (ND)-90.9, ND-64.1, 
ND-96.7 and ND-95.0 ng/ml, respectively. The average recoveries of the DAP analysis method ranged from 93.64 to $99.92 \%$ at DAP concentrations of 10 and $75 \mathrm{ng} / \mathrm{ml}$. The between-day assay coefficients of variation were in the range of 0.59 to $6.45 \%$. The quality control urine samples containing the four DAP metabolites (10 and $75 \mathrm{ng} / \mathrm{ml}$ ) were analyzed together with urine samples. The detection limits of the four DAPs were analyzed according to the methods of the National Institute for Occupational Safety and Health $(\mathrm{NIOSH})^{12)}$. The detection limits of DMP, DEP, DETP and DEDTP in urine were 5.00, 0.034, 0.028 and $0.054 \mathrm{ng} / \mathrm{ml}$. Concentrations were reported in $\mathrm{nmol} / l$, and DEP, DETP and DEDTP were summed as total DEP concentration. Adding DMP to the total DEP resulted in four DAP concentrations. Values below the limit of detection were substituted with zero; the detection limits of DEP, DETP and DEDTP were almost zero ${ }^{13)}$.

All urine samples were analyzed for creatinine by automated clinical chemistry analyzers (Hitachi 917 analyzer SN.0967-09, Japan). The creatinine-adjusted DAP concentration was calculated as nmole/g creatinine. The comparisons of urinary organophosphate metabolite levels used the creatinine-adjusted organophosphate metabolite concentrations due to different quantities of urine excreted in spot urine samples.

\section{Classification of the agricultural worker group}

The pregnant women were classified in to three groups: (G1) higher risk agricultural workers referred to pregnant women who visited the farmland on 4-7 days in the first trimester and at least 2-3 days in the second trimester; $(\mathrm{G} 2)$ moderate/lower risk agricultural workers referred to pregnant women who visited farmland less or had family members in the same house working in a field; and (G3) no agricultural work referred to pregnant women who did not perform work related to an agricultural field.

\section{Data analysis}

The descriptive statistics were calculated using SPSS (version18; PASW Statistics Base 18, Serial no. 5082368, ID no. 5071846) from SPSS (Thailand) Co., Ltd., Thailand. Since exposures were highly skewed, concentrations were reported as the median, range, and interquartile range (IQR). For comparisons, nonparametric analyses were used including the Mann Whitney U test, Kruskal-Wallis test and all pairwise comparisons (Dwass-Steel-Chritchlow-Fligner).

\section{Results}

\section{Demographic characteristics}

Urine samples were collected from 86 women at 28 weeks of pregnancy, 67 at delivery and 51 at
2 months postpartum. The eighty-six 28 weeks pregnant women were 26 years old on average (SD 4.2). Eighty-six percent had some education at the secondary school or high school level. Their occupations included agriculturist (27\%), housewife (20\%) and self-employed (13\%). Agriculturists grew rice, corn, vegetables, flowers, etc. In their homes, $62.8 \%$ of family members of pregnant women worked in agricultural fields. The planting season differed depending on the types of plants. There was at least one growing season at the farm; the growing seasons differ depending on the types of plants. Only one pregnant women smoked cigarettes; she smoked two cigarettes/week. Five pregnant women reported drinking beer; one drank 2-4 times/week, and four drank less than once a month.

Agricultural activities of women at 28 weeks of pregnancy

The pesticide exposures information collected by the questionnaires at 28 weeks of pregnancy and 2 months postpartum is shown in Table 1. During pregnancy, $62 \%$ of pregnant women reported using insecticide in their homes. Of those using insecticides, $72 \%$ applied the insecticide in their homes, and the most common brands reported were Bigon (55\%), Shieldtox (9\%), Aswin (7\%), Art and Shell (5\%) and $16 \%$ were other brands. Forty-five percent of the women reported living near farmlands that were sprayed with pesticides.

The pregnant women were classified by the amount of time they reported working in agricultural fields during pregnancy. Group $1(22 \%)$ was presumed to be at higher risk. Group $2(42 \%)$ was presumed to be at moderate to low risk. Group $3(36 \%)$ had no agricultural work performed by the women or family members.

Pregnant women performed several agricultural activities; they grew plants (23.3\%), applied chemical fertilizer or manure or compost $(19.2 \%)$, applied pesticide $(10.5 \%)$, eliminated weeds $(9.0 \%)$ and handpicked crops, or plants or flowers (24.4\%).

\section{Agricultural activities of women at 2 months postpar- tum}

At 2 months postpartum, $55 \%$ of the women reported using and applying insecticides in their homes. Fifty-one percent reported living near agricultural areas sprayed with pesticides, and $22 \%$ worked outside their home. In Thailand, mothers who deliver infants can take a leave from work for 45 days to take care of their infants at home. The postpartum mothers reported fewer agricultural activities: 2\% grew plants, $8 \%$ applied chemical fertilizer, $2 \%$ eliminated weeds, and $2 \%$ hand-picked crops, plants or flowers. 
Table 1. Agricultural activities of women at 28 weeks of pregnancy and 2 months postpartum

\begin{tabular}{|c|c|c|}
\hline \multirow[t]{2}{*}{ Activities of pregnant women } & $\begin{array}{c}28 \text { weeks of } \\
\text { pregnancy } \\
(n=86)\end{array}$ & $\begin{array}{c}2 \text { months } \\
\text { postpartum } \\
(\mathrm{n}=51)\end{array}$ \\
\hline & Number $(\%)$ & Number $(\%)$ \\
\hline \multicolumn{3}{|l|}{ Application of insecticide in your home } \\
\hline Yes & $53(61.6)$ & $28(54.9)$ \\
\hline No & $33(38.4)$ & $22(43.1)$ \\
\hline \multicolumn{3}{|l|}{ You applied insecticide in your home } \\
\hline Yes & $38(44.2)$ & $28(54.9)$ \\
\hline Never & $48(55.8)$ & $22(43.1)$ \\
\hline \multicolumn{3}{|l|}{ You live next to farmland where pesticides are sprayed } \\
\hline Not near farmland & $43(50.0)$ & $24(47.1)$ \\
\hline Near farmland, don't know if pesticides are sprayed & $4(4.7)$ & $1(2.0)$ \\
\hline Near farmland where pesticide are sprayed & $39(45.3)$ & $26(51.0)$ \\
\hline \multicolumn{3}{|l|}{ How often you visit agricultural fields } \\
\hline Group 1 Agricultural work (Higher risk) & $19(22.1)$ & $13(21.7)$ \\
\hline Group 2 Agricultural work (Moderate/low risk) & $36(41.9)$ & $24(24.5)$ \\
\hline Group 3 No Agricultural work & $31(36.1)$ & $14(33.2)$ \\
\hline \multicolumn{3}{|l|}{ Working outside your home } \\
\hline Yes & $41(47.7)$ & $11(21.6)$ \\
\hline No & $44(51.2)$ & $39(76.5)$ \\
\hline \multicolumn{3}{|l|}{ Your occupation } \\
\hline Agriculturist & $23(27.1)$ & $9(17.6)$ \\
\hline Other occupation & $62(72.9)$ & $41(80.4)$ \\
\hline \multicolumn{3}{|l|}{ You do farm work related to growing plants } \\
\hline Never & $58(67.4)$ & $49(96.1)$ \\
\hline Yes & $20(23.3)$ & $1(2.0)$ \\
\hline \multicolumn{3}{|l|}{ You apply chemical fertilizer, manure or compost } \\
\hline Never & $63(80.8)$ & $46(90.2)$ \\
\hline Yes & $15(19.2)$ & $4(7.8)$ \\
\hline \multicolumn{3}{|l|}{ Applied pesticides } \\
\hline Never & $69(80.2)$ & $50(98.0)$ \\
\hline Yes & $9(10.5)$ & None \\
\hline \multicolumn{3}{|l|}{ You apply herbicide to control weeds } \\
\hline Never & $71(91.0)$ & $48(94.1)$ \\
\hline Yes & $7(9.0)$ & $1(2.0)$ \\
\hline \multicolumn{3}{|l|}{ Hand-pick crops, plants or flowers } \\
\hline Never & $57(66.3)$ & $49(96.0)$ \\
\hline Yes & $21(24.4)$ & $1(2.0)$ \\
\hline
\end{tabular}

\section{Urinary DAP metabolites}

The unadjusted and creatinine-adjusted urinary DAP metabolites at the $28^{\text {th }}$ week of pregnancy, delivery and 2 months postpartum show variability in exposures over time by metabolite (Table 2). For the creatinine-adjusted DAP, the creatinine levels in urine were very low in a few subjects during the delivery period, but the creatinine levels went back to the normal range at 2 months postpartum. The detection frequency of DMP decreased slightly from $78 \%$ at 28 weeks of pregnancy to $72 \%$ at delivery and then to $67 \%$ at 2 months postpartum. The DEP metabolites were detected in 67,72 and $63 \%$ of the urine samples at 28 weeks of pregnancy, delivery and 2 months postpartum, respectively.

Significant differences were found for the median creatinine-adjusted DMP at 28 weeks of pregnancy (48.9 nmole/g) vs. 2 months postpartum $(25.6 \mathrm{nmole} / \mathrm{g}$; 
Table 2. Unadjusted and creatinine-adjusted urinary dialkyl phosphate (DAP) metabolite levels at three time points: 28 weeks of pregnancy, delivery and 2 months postpartum

\begin{tabular}{|c|c|c|c|c|c|}
\hline & \multirow{2}{*}{$\begin{array}{l}\text { Detection } \\
\text { frequency } \\
\quad(\%)\end{array}$} & \multicolumn{2}{|c|}{$\begin{array}{l}\text { Unadjusted DAP } \\
\quad(\mathrm{nmole} / l)\end{array}$} & \multicolumn{2}{|c|}{$\begin{array}{l}\text { Creatinine-adjusted DAP } \\
\text { (nmole/g creatinine) }\end{array}$} \\
\hline & & $\begin{array}{c}50^{\text {th }} \\
\text { percentile }\end{array}$ & Range & $\begin{array}{c}50^{\text {th }} \\
\text { percentile }\end{array}$ & Range \\
\hline \multicolumn{6}{|c|}{28 weeks of pregnancy $(n=86)$} \\
\hline DMP & 77.9 & 37.7 & ND-121.5 & 48.9 & ND-450.9 \\
\hline DEP & 67.4 & 14.6 & ND-72.0 & 20.8 & ND-325.7 \\
\hline DETP & 47.7 & ND & ND-464.4 & ND & ND-956.3 \\
\hline DEDTP & 44.2 & ND & ND-476.8 & ND & ND-1199.5 \\
\hline Total DEP & 89.5 & 45.2 & ND-613.2 & 84.1 & ND-1332.9 \\
\hline DAPs & 95.4 & 90.1 & ND-688.8 & 160.9 & ND-1783.7 \\
\hline \multicolumn{6}{|c|}{ At delivery $(n=67)$} \\
\hline DMP & 71.6 & 36.4 & ND-166.0 & 50.5 & ND-829.8 \\
\hline DEP & 71.6 & 14.4 & ND-102.8 & 18.8 & ND-376.0 \\
\hline DETP & 52.2 & 2.4 & ND-100.5 & 6.5 & ND-755.8 \\
\hline DEDTP & 53.7 & 2.3 & ND-505.4 & 4.5 & ND-2066.2 \\
\hline Total DEP & 94.0 & 64.9 & $8.1-569.8$ & 87.4 & ND-2066.2 \\
\hline DAPs & 100.0 & 108.9 & $11.0-597.2$ & 190.8 & ND-2442.8 \\
\hline \multicolumn{6}{|c|}{ 2-month postpartum $(\mathrm{n}=51)$} \\
\hline DMP & 66.7 & 38.0 & ND-720.3 & 25.6 & ND-637.3 \\
\hline DEP & 62.8 & 17.3 & ND-416.2 & 14.8 & ND-743.3 \\
\hline DETP & 41.2 & ND & ND-301.3 & ND & ND-240.8 \\
\hline DEDTP & 49.0 & ND & ND-510.0 & ND & ND-1399.2 \\
\hline Total DEP & 80.4 & 46.7 & ND-717.4 & 56.1 & ND-1546.4 \\
\hline DAPs & 90.2 & 92.1 & ND-805.1 & 101.8 & ND-1546.4 \\
\hline
\end{tabular}

DMPs: dimethyl phosphate. DEP: diethyl phosphate. DETP: diethyl thiophosphate. DEDTP: diethyl dithiophosphate, ND: not detectable.

at $p=0.011)$ and the median creatinine-adjusted total DEP at delivery $(87.4 \mathrm{nmole} / \mathrm{g})$ vs. 2 months postpartum $(56.1 \mathrm{nmole} / \mathrm{g}$; at $p=0.027)$. There were also a significant differences for the median creatinine-adjusted DAPs at 28 weeks of pregnancy $(160.9 \mathrm{nmole} / \mathrm{g})$ vs. 2 months postpartum (101.8 nmole/g) and delivery $(190.8 \mathrm{nmole} / \mathrm{g})$ vs. 2 months postpartum (101.8 nmole/g; $p=0.047$ and 0.013 , respectively).

\section{Pesticide exposure at their homes}

The urinary DMP and DAP concentrations of pregnant women who reported that they lived near agricultural areas sprayed with pesticides were significantly higher urine concentrations than those that did not in analyses with the Kruskal-Wallis test and all pairwise comparisons (Dwass-Steel-Chritchlow-Fligner; $p=0.011$ and 0.021 , respectively; Table 3).

\section{Agricultural work risk groups}

Regarding the agricultural work groups in the women at 28 weeks of pregnancy, Group 1 (higher risk agricultural workers) had a higher total DEP concentration in their urine compared with Group 2 (moderate/low risk agricultural workers, $p=0.043$ ) and Group 3 (no agricultural work, $p=0.021$ ) in analyses with the Kruskal-Wallis test and all pairwise comparisons (Dwass-Steel-Chritchlow-Fligner) (Table 4). Women in Group 1 (higher risk agricultural workers) also had concentrations of DAPs higher in their urine compared with Group 2 (moderate/low risk agricultural workers, $p=0.033$ ) and Group 3 (no agricultural work, $p=0.019$ ) (Table 4).

Pesticide exposure from agricultural work activities in the 28th week of pregnancy

Those who worked outside their homes had significantly higher urinary total concentrations of DEP and DAPs than those worked at home (Mann-Whitney $\mathrm{U}$ test $p=0.030$ and 0.015 , respectively) (Table 5). Pregnant women who reported their occupation as agriculturists had significantly higher urinary DEDTP concentrations than those working in other occupations (Mann-Whitney U test $p=0.047$ ). Pregnant women who grew plants, applied chemical fertilizer, 
Table 3. Creatinine-adjusted urinary organophosphate metabolites (nmole/g) of women in the $28^{\text {th }}$ week of pregnancy categorized by potential for home exposure to pesticides from nearby farmland

\begin{tabular}{|c|c|c|c|c|c|c|c|}
\hline Parameter & & DMP & DEP & DETP & DEDTP & Total DEP & DAPs \\
\hline \multicolumn{8}{|l|}{ Do you live next to farmland? } \\
\hline C1. Not near farmland $(n=43)$ & $\begin{array}{l}\text { Median } \\
(\mathrm{IQR})^{\mathrm{a}}\end{array}$ & $\begin{array}{l}37.6 \\
\left(N^{b}-78.1\right)\end{array}$ & $\begin{array}{l}16.3 \\
(\mathrm{ND}-38.9)\end{array}$ & $\begin{array}{l}\mathrm{ND} \\
(\mathrm{ND}-38.9)\end{array}$ & $\begin{array}{l}\mathrm{ND} \\
(\mathrm{ND}-22.7)\end{array}$ & $\begin{array}{l}70.6 \\
(16.3-148.5)\end{array}$ & $\begin{array}{l}108.7 \\
(49.1-235.4)\end{array}$ \\
\hline $\begin{array}{l}\text { C2. Near farmland but don't know } \\
\text { if pesticides are sprayed }(n=4)\end{array}$ & $\begin{array}{c}\text { Median } \\
(\mathrm{IQR})\end{array}$ & $\begin{array}{l}88.5 \\
(55.8-126.9)\end{array}$ & $\begin{array}{l}39.5 \\
(5.2-67.2)\end{array}$ & $\begin{array}{l}54.7 \\
(\mathrm{ND}-139.1)\end{array}$ & $\begin{array}{l}\mathrm{ND} \\
(\mathrm{ND}-5.7)\end{array}$ & $\begin{array}{l}113.4 \\
(33.1-164.8)\end{array}$ & $\begin{array}{l}201.9 \\
(88.9-291.7)\end{array}$ \\
\hline $\begin{array}{l}\text { C3. Near farmland where } \\
\text { pesti cides are sprayed }(n=39)\end{array}$ & $\begin{array}{l}\text { Median } \\
(\mathrm{IQR})\end{array}$ & $\begin{array}{l}67.0 \\
(40.3-124.7)\end{array}$ & $\begin{array}{l}30.9 \\
(9.6-60.3)\end{array}$ & $\begin{array}{l}15.0 \\
(\mathrm{ND}-70.2)\end{array}$ & $\begin{array}{l}1.5 \\
(\mathrm{ND}-34.4)\end{array}$ & $\begin{array}{l}94.3 \\
(47.2-243.5)\end{array}$ & $\begin{array}{l}202.1 \\
(94.9-317.8)\end{array}$ \\
\hline $\begin{array}{l}p \text {-value for Kruskal-Wallis test } \\
\text { and all pairwise comparisons } \\
\text { (Dwass-Steel-Chritchlow-Fligner) }\end{array}$ & & $\begin{array}{l}0.006^{*} \\
\text { C1 vs. } C 2=0.128 \\
\text { C1 vs. } C 3=0.011^{*} \\
\text { C2 vs. } C 3=0.680\end{array}$ & 0.106 & 0.629 & 0.352 & 0.148 & $\begin{array}{l}0.026^{*} \\
\mathrm{C} 1 \text { vs. } \mathrm{C} 2=0.631 \\
\mathrm{C} 1 \text { vs. } \mathrm{C} 3=0.021^{*} \\
\mathrm{C} 2 \text { vs. } \mathrm{C} 3=0.890\end{array}$ \\
\hline
\end{tabular}

${ }^{a}$ IQR: interquartile range. ${ }^{b} \mathrm{ND}$ : not detectable. DMP: dimethyl phosphate. DEP: diethyl phosphate. DETP: diethyl thiophosphate. DEDTP: diethyl dithiophosphate, DAP: dialkyl phosphate. *significant at $p<0.05$.

Table 4. Creatinine-adjusted urinary organophosphate metabolites (DMP, DEP, DETP, DEDTP, Total DEP and DAPs in nmole/g) of 28 weeks of pregnant women from agricultural field visits during the first and second trimester of pregnancy

\begin{tabular}{|c|c|c|c|c|c|c|c|}
\hline Parameter & & DMP & DEP & DETP & DEDTP & Total DEP & DAPs \\
\hline \multicolumn{8}{|l|}{ How often you visit the farmland? } \\
\hline $\begin{array}{l}\text { Group } 1(\mathrm{G} 1) \text { Higher risk agricul- } \\
\text { tural worker }(\mathrm{n}=19)\end{array}$ & $\begin{array}{l}\text { Median } \\
(\mathrm{IQR})^{\mathrm{a}}\end{array}$ & $\begin{array}{l}59.3 \\
(36.0-125.9)\end{array}$ & $\begin{array}{l}21.7 \\
(13.5-63.1)\end{array}$ & $\begin{array}{l}21.4 \\
(0-138.6)\end{array}$ & $\begin{array}{l}\mathrm{ND}^{\mathrm{b}} \\
(\mathrm{ND}-128.5)\end{array}$ & $\begin{array}{l}201.7 \\
(60.6-268.1)\end{array}$ & $\begin{array}{l}292.6 \\
(91.3-346.2)\end{array}$ \\
\hline $\begin{array}{l}\text { Group } 2(\mathrm{G} 2) \text { Moderate/ lower } \\
\text { risk agricultural workers }(\mathrm{n}=36)\end{array}$ & $\begin{array}{l}\text { Median } \\
(\mathrm{IQR})\end{array}$ & $\begin{array}{l}46.2 \\
(\mathrm{ND}-98.4)\end{array}$ & $\begin{array}{l}20.0 \\
(\mathrm{ND}-44.8)\end{array}$ & $\begin{array}{l}\text { ND } \\
(\mathrm{ND}-67.7)\end{array}$ & $\begin{array}{l}0.8 \\
(\mathrm{ND}-22.7)\end{array}$ & $\begin{array}{l}87.6 \\
(27.0-167.4)\end{array}$ & $\begin{array}{l}162.4 \\
(82.6-273.0)\end{array}$ \\
\hline $\begin{array}{l}\text { Group } 3 \text { (G3) No agricultural } \\
\text { work }(\mathrm{n}=31)\end{array}$ & $\begin{array}{l}\text { Median } \\
(\mathrm{IQR})\end{array}$ & $\begin{array}{l}48.7 \\
(22.7-98.4)\end{array}$ & $\begin{array}{l}20.8 \\
(\mathrm{ND}-41.5)\end{array}$ & $\begin{array}{l}\mathrm{ND} \\
(0-34.9)\end{array}$ & $\begin{array}{l}\mathrm{ND} \\
(\mathrm{ND}-22.7)\end{array}$ & $\begin{array}{l}65.6 \\
(17.1-120.0)\end{array}$ & $\begin{array}{l}126.3 \\
(65.2-198.4)\end{array}$ \\
\hline $\begin{array}{l}p \text {-value for Kruskal-Wallis test } \\
\text { and all pairwise comparisons } \\
\text { (Dwass-Steel-Chritchlow-Fligner) }\end{array}$ & & 0.527 & 0.245 & 0.240 & 0.596 & $\begin{array}{l}0.027^{*} \\
\mathrm{G} 1 \text { vs. } \mathrm{G} 2=0.043^{*} \\
\mathrm{G} 1 \text { vs. } \mathrm{G} 3=0.021^{*} \\
\mathrm{G} 2 \text { vs. } \mathrm{G} 3=0.968\end{array}$ & $\begin{array}{l}0.021 * \\
\mathrm{G} 1 \text { vs. } \mathrm{G} 2=0.033^{*} \\
\mathrm{G} 1 \text { vs. } \mathrm{G} 3=0.019^{*} \\
\mathrm{G} 2 \text { vs. } \mathrm{G} 3=0.990\end{array}$ \\
\hline
\end{tabular}

${ }^{\mathrm{a}}$ IQR: interquartile range. ${ }^{\mathrm{b}} \mathrm{ND}$ : not detectable. DMP: dimethyl phosphate. DEP: diethyl phosphate. DETP: diethyl thiophosphate. DEDTP: diethyl dithiophosphate, DAP: dialkyl phosphate. *significant at $p<0.05$.

applied pesticide, eliminated weeds and picked crops/ plants/flowers by hand had significantly higher urinary pesticide metabolites (DEDTP, total DEP or DAPs) than those who did not $(p<0.05)$ (Table 5).

\section{Pesticide exposure from agricultural work activities at 2 months postpartum}

The urinary DETP metabolite concentrations of the women who reported their occupation as agriculturist were significantly higher than those who reported other occupations (Mann-Whitney U test $p=0.013$ ) (Table 6). Women who reported agricultural activities at 2 months postpartum had significantly higher DAP concentrations than those that did not (Mann-Whitney $\mathrm{U}$ test $p=0.027$ ).

\section{Discussion}

In our cohort, $27 \%$ of the women reported their occupation as agriculturist, and more than half of them had family members in the same house who worked in agriculture. These women may help with some of the agricultural activities both at home and in the farm fields. Some of them grew plants for family use and to sell. Some of these women may be exposed to pesticides at home, since they reported using insecticides at their homes and some lived near agricultural fields where pesticides were sprayed.

Regarding creatinine-unadjusted urinary metabolites, the organophosphate metabolites DMP, DEP, DETP, DEDTP, and total DEP and DAPs were not significantly different when comparing women at 28 weeks of pregnancy, delivery and 2 months postpartum. Although the women reduced their agricultural activities after giving birth, the unadjusted-urinary metabolites tended to slightly increase at 2 months postpartum. Bradman et al. also found an upward shift of 
Table 5. Creatinine-adjusted urinary organophosphate metabolites (nmole/g) from agricultural activities among women in the $28^{\text {th }}$ week of pregnancy

\begin{tabular}{|c|c|c|c|c|c|c|c|}
\hline Parameter & & DMP & DEP & DETP & DEDTP & Total DEP & DAPs \\
\hline \multicolumn{8}{|c|}{ Do you work outside your home? } \\
\hline No $(n=44)$ & $\begin{array}{l}\text { Median } \\
(\mathrm{IQR})^{\mathrm{a}}\end{array}$ & $\begin{array}{l}49.6 \\
\left(N D^{b-96.9)}\right.\end{array}$ & $\begin{array}{l}20.4 \\
(\mathrm{ND}-50.5)\end{array}$ & $\begin{array}{l}\text { ND } \\
(N D-49.0)\end{array}$ & $\begin{array}{l}\mathrm{ND} \\
(\mathrm{ND}-15.1)\end{array}$ & $\begin{array}{l}61.7 \\
(16.1-150.8)\end{array}$ & $\begin{array}{l}105.7 \\
(54.4-243.1)\end{array}$ \\
\hline Yes $(n=41)$ & $\begin{array}{l}\text { Median } \\
(\mathrm{IQR})\end{array}$ & $\begin{array}{l}48.3 \\
(33.9-104.4)\end{array}$ & $\begin{array}{l}20.8 \\
(\mathrm{ND}-46.0)\end{array}$ & $\begin{array}{l}21.4 \\
(\mathrm{ND}-75.8)\end{array}$ & $\begin{array}{l}2.5 \\
(\mathrm{ND}-42.6)\end{array}$ & $\begin{array}{l}117.5 \\
(51.5-234.1)\end{array}$ & $\begin{array}{l}195.3 \\
(105.1-323.2)\end{array}$ \\
\hline$p$-value & & 0.519 & 0.957 & 0.135 & 0.068 & $0.030^{*}$ & $0.015^{*}$ \\
\hline \multicolumn{8}{|c|}{ What is your occupation? } \\
\hline $\begin{array}{l}\text { Agriculturist } \\
(\mathrm{n}=22)\end{array}$ & $\begin{array}{l}\text { Median } \\
(\mathrm{IQR})\end{array}$ & $\begin{array}{l}50.5 \\
(26.4-129.8)\end{array}$ & $\begin{array}{l}23.4 \\
(9.8-60.7)\end{array}$ & $\begin{array}{l}\mathrm{ND} \\
(\mathrm{ND}-64.0)\end{array}$ & $\begin{array}{l}15.2 \\
(0-147)\end{array}$ & $\begin{array}{l}113.9 \\
(31.9-266.1)\end{array}$ & $\begin{array}{l}281.2 \\
(80.5-351.5)\end{array}$ \\
\hline $\begin{array}{l}\text { Other occupation } \\
(\mathrm{n}=62)\end{array}$ & $\begin{array}{l}\text { Median } \\
\text { (IQR) }\end{array}$ & $\begin{array}{l}49.6 \\
(17.1-97.8)\end{array}$ & $\begin{array}{l}20.8 \\
(\mathrm{ND}-44.2)\end{array}$ & $\begin{array}{l}\text { ND } \\
(\mathrm{ND}-57.9)\end{array}$ & $\begin{array}{l}\text { ND } \\
\text { (ND-15.0) }\end{array}$ & $\begin{array}{l}70.4 \\
(20.6-156.6)\end{array}$ & $\begin{array}{l}148.0 \\
(68.9-246.7)\end{array}$ \\
\hline$p$-value & & 0.465 & 0.287 & 0.956 & $0.047^{*}$ & 0.196 & 0.100 \\
\hline \multicolumn{8}{|c|}{ During your pregnancy, did you do any of the following agricultural work $\cdots$ grow plants? } \\
\hline Never $(\mathrm{n}=58)$ & $\begin{array}{l}\text { Median } \\
(\mathrm{IQR})\end{array}$ & $\begin{array}{l}49.6 \\
(23.7-98.5)\end{array}$ & $\begin{array}{l}20.9 \\
(\mathrm{ND}-44.2)\end{array}$ & $\begin{array}{l}\mathrm{ND} \\
(\mathrm{ND}-37.8)\end{array}$ & $\begin{array}{l}\text { ND } \\
\text { (ND-16.7) }\end{array}$ & $\begin{array}{l}75.8 \\
(24.1-156.6)\end{array}$ & $\begin{array}{l}150.0 \\
(75.2-242.6)\end{array}$ \\
\hline Yes $(n=20)$ & $\begin{array}{l}\text { Median } \\
(\mathrm{IQR})\end{array}$ & $\begin{array}{l}56.2 \\
(37.0-136.6)\end{array}$ & $\begin{array}{l}20.8 \\
(2.9-62.8)\end{array}$ & $\begin{array}{l}10.7 \\
(0-120.6)\end{array}$ & $\begin{array}{l}18.7 \\
(0-144.7)\end{array}$ & $\begin{array}{l}183.5 \\
(50.9-270.4)\end{array}$ & $\begin{array}{l}281.2 \\
(119.0-337.1)\end{array}$ \\
\hline$p$-value & & 0.275 & 0.519 & 0.360 & 0.095 & 0.078 & $0.025^{*}$ \\
\hline \multicolumn{8}{|c|}{ During your pregnancy, did you do any of the following agricultural work $\cdots$ apply chemical fertilizer, manure or compost? } \\
\hline Never $(n=63)$ & $\begin{array}{l}\text { Median } \\
(\mathrm{IQR})\end{array}$ & $\begin{array}{l}50.2 \\
(22.7-98.7)\end{array}$ & $\begin{array}{l}20.9 \\
(\mathrm{ND}-46.0)\end{array}$ & $\begin{array}{l}\text { ND } \\
(\mathrm{ND}-43.7)\end{array}$ & $\begin{array}{l}\text { ND } \\
\text { (ND-16.6) }\end{array}$ & $\begin{array}{l}81.1 \\
(21.7-156.7)\end{array}$ & $\begin{array}{l}156.7 \\
(75.1-243.9)\end{array}$ \\
\hline Yes $(n=15)$ & $\begin{array}{l}\text { Median } \\
(\mathrm{IQR})\end{array}$ & $\begin{array}{l}59.4 \\
(44.1-135.1)\end{array}$ & $\begin{array}{l}16.6 \\
(\mathrm{ND}-51.6)\end{array}$ & $\begin{array}{l}16.5 \\
\text { (ND-138.6) }\end{array}$ & $\begin{array}{l}24.5 \\
(0-202.5)\end{array}$ & $\begin{array}{l}201.7 \\
(57.8-272.0)\end{array}$ & $\begin{array}{l}292.6 \\
(95.6-367.4)\end{array}$ \\
\hline$p$-value & & 0.184 & 0.862 & 0.320 & $0.043 *$ & $0.033^{*}$ & $0.010^{*}$ \\
\hline \multicolumn{8}{|c|}{ During your pregnancy, did you do any of the following agricultural work $\cdots$ apply pesticides? } \\
\hline Never $(n=69)$ & $\begin{array}{l}\text { Median } \\
\text { (IQR) }\end{array}$ & $\begin{array}{l}50.2 \\
(25.6-98.6)\end{array}$ & $\begin{array}{l}20.9 \\
(\mathrm{ND}-45.5)\end{array}$ & $\begin{array}{l}\text { ND } \\
(\mathrm{ND}-49.4)\end{array}$ & $\begin{array}{l}\text { ND } \\
\text { (ND-16.8) }\end{array}$ & $\begin{array}{l}87.0 \\
(23.3-166.8)\end{array}$ & $\begin{array}{l}159.1 \\
(9.3-164.4)\end{array}$ \\
\hline Yes $(n=9)$ & $\begin{array}{l}\text { Median } \\
(\mathrm{IQR})\end{array}$ & $\begin{array}{l}59.4 \\
(44.9-218.5)\end{array}$ & $\begin{array}{l}39.4 \\
(6.76-57.3)\end{array}$ & $\begin{array}{l}21.4 \\
(\mathrm{ND}-389.1)\end{array}$ & $\begin{array}{l}30.6 \\
(12.6-126.7)\end{array}$ & $\begin{array}{l}201.7 \\
(61.3-467.2)\end{array}$ & $\begin{array}{l}311.5 \\
(183.9-544.6)\end{array}$ \\
\hline$p$-value & & 0.142 & 0.460 & 0.306 & $0.009 *$ & 0.062 & $0.023^{*}$ \\
\hline \multicolumn{8}{|c|}{ During your pregnancy, did you do any of the following agricultural work... eliminate weeds? } \\
\hline Never $(\mathrm{n}=71)$ & $\begin{array}{l}\text { Median } \\
\text { (IQR) }\end{array}$ & $\begin{array}{l}50.2 \\
(27.2-101.5)\end{array}$ & $\begin{array}{l}20.9 \\
(\mathrm{ND}-46.0)\end{array}$ & $\begin{array}{l}\text { ND } \\
(\mathrm{ND}-43.7)\end{array}$ & $\begin{array}{l}\text { ND } \\
\text { (ND-22.7) }\end{array}$ & $\begin{array}{l}81.1 \\
(24.87-165.3)\end{array}$ & $\begin{array}{l}159.1 \\
(83.4-278.9)\end{array}$ \\
\hline Yes $(n=7)$ & $\begin{array}{l}\text { Median } \\
\text { (IQR) }\end{array}$ & $\begin{array}{l}59.4 \\
(47.0-109.9)\end{array}$ & $\begin{array}{l}39.4 \\
(\mathrm{ND}-63.1)\end{array}$ & $\begin{array}{l}70.2 \\
(\mathrm{ND}-138.6)\end{array}$ & $\begin{array}{l}24.5 \\
(\mathrm{ND}-202.5)\end{array}$ & $\begin{array}{l}206.7 \\
(140.7-374.5)\end{array}$ & $\begin{array}{l}285.9 \\
(273.6-421.5)\end{array}$ \\
\hline$p$-value & & 0.511 & 0.677 & 0.245 & 0.101 & $0.034^{*}$ & $0.038^{*}$ \\
\hline \multicolumn{8}{|c|}{ During your pregnancy, did you do any of the following agricultural work $\cdots$. hand-pick crops/plants/flowers? } \\
\hline Never $(n=57)$ & $\begin{array}{l}\text { Median } \\
\text { (IQR) }\end{array}$ & $\begin{array}{l}48.7 \\
(25.0-98.6)\end{array}$ & $\begin{array}{l}20.9 \\
(\mathrm{ND}-44.5)\end{array}$ & $\begin{array}{l}\text { ND } \\
\text { (ND-49.4) }\end{array}$ & $\begin{array}{l}\text { ND } \\
(\mathrm{ND}-20.0)\end{array}$ & $\begin{array}{l}87.0 \\
(23.3-156.6)\end{array}$ & $\begin{array}{l}156.7 \\
(78.7-243.0)\end{array}$ \\
\hline Yes $(n=21)$ & $\begin{array}{l}\text { Median } \\
\text { (IQR) }\end{array}$ & $\begin{array}{l}59.4 \\
(41.0-130.7)\end{array}$ & $\begin{array}{l}17.9 \\
(6.5-56.8)\end{array}$ & $\begin{array}{l}20.6 \\
(\mathrm{ND}-136.1)\end{array}$ & $\begin{array}{l}2.5 \\
(\mathrm{ND}-89.7)\end{array}$ & $\begin{array}{l}165.3 \\
(52.5-281.4)\end{array}$ & $\begin{array}{l}291.1 \\
(88.4-373.6)\end{array}$ \\
\hline$p$-value & & 0.188 & 0.457 & 0.225 & 0.297 & 0.082 & $0.019^{*}$ \\
\hline
\end{tabular}

${ }^{\mathrm{a}}$ IQR: interquartile range. ${ }^{\mathrm{b}} \mathrm{ND}$ : not detectable. DMP: dimethyl phosphate. DEP: diethyl phosphate. DETP: diethyl thiophosphate. DEDTP: diethyl dithiophosphate. Total DEP=DEP + DETP + DEDTP. DAPs=DMP + Total DEP. *significant at $p<0.05$.

DAP metabolites after delivery (9 days postpartum) compared with prenatal levels. The urinary total DAP levels in the prenatal period in the current study were similar to those in the study of Bradman et al., but the urinary DAP levels at 2 months postpartum were considerably lower than those in the study of
Bradman et al. at 9 days postpartum ${ }^{7}$. The urinary DMP levels in the current study were much higher than those in the study of Bradman et al. ${ }^{7}$. This may be because Thai people apply more insecticides to prevent mosquitoes and insects at home. The insecticides used in the home (such as ARS, Shell, Bigon 
Table 6. Creatinine-adjusted urinary organophosphate metabolites (nmole/g) from agricultural activities among women at 2 months postpartum

\begin{tabular}{|c|c|c|c|c|c|c|c|}
\hline Parameter & & DMP & DEP & DETP & DEDTP & Total DEP & DAPs \\
\hline \multicolumn{8}{|c|}{ Since your baby was born, what is your occupation? } \\
\hline Agriculturist $(\mathrm{n}=9)$ & $\begin{array}{l}\text { Median } \\
(\mathrm{IQR})^{\mathrm{a}}\end{array}$ & $\begin{array}{l}27.9 \\
\left(\mathrm{ND}^{\mathrm{b}}-91.9\right)\end{array}$ & $\begin{array}{l}4.4 \\
(0-22.6)\end{array}$ & $\begin{array}{l}24.8 \\
(5.1-62.5)\end{array}$ & $\begin{array}{l}11.1 \\
(\mathrm{ND}-180.7)\end{array}$ & $\begin{array}{l}64.7 \\
(27.0-247.9)\end{array}$ & $\begin{array}{l}195.3 \\
(76.4-293.2)\end{array}$ \\
\hline Other occupation $(\mathrm{n}=41)$ & $\begin{array}{l}\text { Median } \\
\text { (IQR) }\end{array}$ & $\begin{array}{l}25.6 \\
(\mathrm{ND}-60.8)\end{array}$ & $\begin{array}{l}15.1 \\
(\mathrm{ND}-39.2)\end{array}$ & $\begin{array}{l}\text { ND } \\
\text { (ND-22.4) }\end{array}$ & $\begin{array}{l}\text { ND } \\
\text { (ND-59.3) }\end{array}$ & $\begin{array}{l}31.7 \\
(5.1-103.6)\end{array}$ & $\begin{array}{l}84.8 \\
(23.0-207.3)\end{array}$ \\
\hline$p$-value & & 0.739 & 0.413 & $0.013^{*}$ & 0.311 & 0.265 & 0.098 \\
\hline \multicolumn{8}{|c|}{ Since your baby was born, did you do any agricultural work? } \\
\hline No $(n=46)$ & $\begin{array}{l}\text { Median } \\
\text { (IQR) }\end{array}$ & $\begin{array}{l}25.6 \\
(\mathrm{ND}-59.1)\end{array}$ & $\begin{array}{l}15.0 \\
(\mathrm{ND}-37.0)\end{array}$ & $\begin{array}{l}\text { ND } \\
(\mathrm{ND}-24.8)\end{array}$ & $\begin{array}{l}\mathrm{ND} \\
\text { (ND-55.4) }\end{array}$ & $\begin{array}{l}37.7 \\
(13.0-102.7)\end{array}$ & $\begin{array}{l}84.7 \\
(36.9-207.2)\end{array}$ \\
\hline Yes $(n=4)$ & $\begin{array}{l}\text { Median } \\
\text { (IQR) }\end{array}$ & $\begin{array}{l}49.2 \\
\text { (ND-248.6) }\end{array}$ & $\begin{array}{l}2.2 \\
(\mathrm{ND}-103.7)\end{array}$ & $\begin{array}{l}30.1 \\
(\mathrm{ND}-103.0)\end{array}$ & $\begin{array}{l}90.8 \\
(2.8-185.8)\end{array}$ & $\begin{array}{l}213.1 \\
(48.8-256.5)\end{array}$ & $\begin{array}{l}264.7 \\
(204.2-347.3)\end{array}$ \\
\hline$p$-value & & 0.689 & 0.607 & 0.381 & 0.251 & 0.290 & $0.027 *$ \\
\hline
\end{tabular}

${ }^{a}$ IQR: interquartile range. ${ }^{b} \mathrm{ND}$ : not detectable. DMP: dimethyl phosphate. DEP: diethyl phosphate. DETP: diethyl thiophosphate. DEDTP: diethyl dithiophosphate. Total DEP=DEP + DETP + DEDTP. DAPs=DMP + Total DEP. *significant at $p<0.05$.

and Shieldtox) contain $0.5 \%$ dichlovos together with pyrethroid or carbamate insecticides ${ }^{14}$. ARS contains $0.2 \%$ permethrin or $0.07 \%$ Neo-Pynamin. Bigon contains $0.3 \%$ tetramethrin or $1 \%$ Propoxur. Sheldtox contains $0.1 \%$ bioallethrin. Dichlorvos can metabolize to DMP in the body ${ }^{15)}$.

Regarding creatinine-adjusted organophosphate metabolites, the DMP and DAPs metabolite concentrations during the prenatal and delivery periods were similar, but the DAPs were significantly lower at 2 months postpartum. This may be the result of the reduced exposure of postpartum mothers or the physical changes postpartum in the mother's metabolism and efficiency of toxin clearance as well as blood volume and body weight ${ }^{16)}$. The observation of higher exposures during the prenatal period may reflect the report by the women that they continued to go to the fields up to delivery, and since the organophosphate metabolites have a half-life of 15-30 hours, this would show up in urine samples ${ }^{17}$. A previous study reported median urinary creatinine-adjusted concentrations of DMP, DEP, DETP and DEDTP in small scale farmers of $\mathrm{ND}, \mathrm{ND}, 0.88 \mu \mathrm{g} / \mathrm{g}$ creatinine and ND, respectively ${ }^{10)}$. The current study reported median urinary creatinine-adjusted concentrations of DMP, DEP, DETP and DEDTP in pregnant women of $48.87 \mathrm{nmole} / \mathrm{g}$ creatinine, $20.82 \mathrm{nmole} / \mathrm{g}$ creatinine, $\mathrm{ND}$ and ND; the exposure of pesticides in pregnant women was probably lower than those in the smallscale farmers.

Regarding those working as agriculturists, there were higher concentrations of metabolites in the DEP group (particularly DEDTP and total DEP), for activities related to farming (Table 5). The types of organophosphate and carbamate pesticides reportedly used on the farms of $48.39 \%$ subjects and family members included profenofos (3.3\%), methyl parathion $(6.6 \%)$, acephate (3.3\%), methomyl (20.0\%), etc.

The results of the current study clearly presented that pregnant women were exposed to organophosphate pesticides and that this was dependent on living close to agricultural fields, use of insecticides to prevent mosquitoes and insects at home, use of pesticides in their work as agriculturists, field visits and various agricultural activities. To reduce organophosphate pesticides exposure, pregnant women and postpartum mothers should reduce pesticide exposure to as low as possible to prevent developmental neurotoxicity in their children.

The limitation of this study was that the numbers of urine samples from women at 28 weeks of pregnancy, delivery and 2 months postpartum were low (86, 67 and 51, respectively); some urine samples were lost at Sawanpracharak Hospital in Nakornsawan Province due to terrible flooding in Thailand last year.

Acknowledgment: The project described was supported by Award Number R21HD060520 from the Eunice Kennedy Shriver National Institute of Child Health and Human Development. The content is solely the responsibility of the authors and does not necessarily represent the official views of the Eunice Kennedy Shriver National Institute of Child Health and Human Development or the National Institutes of Health. We would like to thank the nurses, doctors, pediatricians and subjects who participated in this project from the three hospitals, Paholpolphayuhasena, Sawanpracharuk and Amnatcharoen Hospitals in Kanchanaburi, Nakhonsawan and Amnatcharoen Provinces, respectively, for their help and excellent 
care of the subjects and their infants.

\section{References}

1) National Statistic Office, Ministry of Information and Communication Technology. The 2010 population and housing census. [Online]. 2010 [cited 2013 Feb 20]; Available from: URL: http://service.nso. go.th/nso/nsopublish/download/files/lfsSum54.pdf

2) Office of Agricultural Economics, Ministry of Agricultural and Cooperatives [Online]. 2010 [cited 2010 Oct 10]; Available from: URL: http://www. oae.go.th/ewt_news.php?nid=146\&filename=index

3) Ten health issues. Falling IQs among Thai children: Time for more educational reform [Online]. 2013 [cited 2013 Apr 1]; Available from: URL: http://www.hiso.or.th/hiso/picture/reportHealth/ ThaiHealth2011/eng2011_14.pdf

4) Young JG, Eskenazi B, Gladstone EA, et al. Association between in utero organophosphate pesticide exposure and abnormal reflexes in neonates. Neurotoxicology 2005; 26: 199-209.

5) Eskenazi B, Marks AR, Bradman A, et al. Organophosphate pesticide exposure and neurodevelopment in young Mexican-American children. Environ Health Perspect 2007; 115: 792-8.

6) Bouchard MF, Chevrier J, Harley KG, et al. Prenatal exposure to organophosphate pesticides and IQ in 7-year old children. Environ Health Perspect 2010; 119: 1189-95.

7) Bradman A, Eskenazi B, Barr DB, et al. Organophosphate urinary metabolite levels during pregnancy and after delivery in women living in an agricultural community. Environ Health Perspect 2005; 113: 1802-7.

8) Rice D, Barone S Jr. Critical periods of vulnerability for the developing nervous system: evidence from humans and animal models. Environ Health Perspect 2000; 108 (Suppl 3): 511-33.

9) Health Systems Research Institute. The situation of pesticide usage in Thailand. Final report; 2005. p. 54 (In Thai).

10) Panuweta P, Prapamontolc $T$, Chantarad S, et al. Concentrations of urinary pesticide metabolites in small-scale farmers in Chiang Mai Province, Thailand. Sci Total Environ 2008; 407: 655-68.

11) Alwis GK, Needham LL, Barr DB. Measurement of human urinary organophosphate pesticide metabolites by automated solid-phase extraction, post extraction derivatization, and gas chromatographytandem mass spectrometry. J Chromatog B Analyt Technol Biomed Life Sci 2006; 843: 34-41.

12) National Institute for Occupational Safety and Health. Limits of detection and quantification. In: Kennedy ER, Fischbach TJ, Song R, Eller PM, Sulman SA, editors. Guidelines for air sampling and analytical method development and evaluation. A NIOSH technical report. Cincinnati $(\mathrm{OH})$ : Department of Health and Human Services; 1994. p.65-8.

13) Barr DB, Landsittel D, Nishioka M, et al. A survey of laboratory and statistical issues related to farmworker exposure studies. Environ Health Perspect 2006; 114; 961-8.

14) Toxicology Information Center, Department of Medical Science, Ministry of Public Health, Thailand. (In Thai) [Online]. 2012 [cited 2012 Oct 1]; Available from: URL: http://webdb.dmsc.moph. go.th/ifc_toxic/a_tx_1_001c.asp?info_id=384

15) Dichorvos, environmental health criteria 79 , International programme on chemical safety. [Online]. 2013 [cited 2013 Mar 28]; Available from: URL: http://www.inchem.org/documents/ehc/ehc/ehc79.htm

16) Cunningham FG, MacDonald PC, Kant NF, Leveno KJ, Gilstrap LC, eds. Williams Obstetrics. 20th ed. Stamford (UK): Appleton \& Lang; 1997.

17) Griffin P, Mason H, Heywood K, Cocker J. Oral and dermal absorption of chlorpyrifos. Occup Environ Med 1999; 56: 10-3. 